\title{
THE EXISTENCE OF SOLUTION OF THE INVERSE SPECTRAL PROBLEM FOR DISCRETE SELF-ADJOINT SEMI-BOUNDED FROM BELOW OPERATOR
}

\author{
G.A. Zakirova ${ }^{1}$, zakirovaga@susu.ac.ru, \\ E.V.Kirillov ${ }^{1}$, kirillovev@susu.ac.ru. \\ ${ }^{1}$ South Ural State University, Chelyabinsk, Russian Federation.
}

Inverse spectral problems have many applications in engineering and physics. It was investigated for a variety of tasks specific operators. In this article explores the inverse spectral problem for abstract discrete self-adjoint semi-bounded from below operator. Using the resolvent method and principle of the contraction mapping theorem of the existence of the inverse problem solution is proved.

Keywords: perturbed operator; discrete self-adjoint operator; potential.

\section{Introduction}

It is difficult to overestimate the role that play inverse spectral problems in mathematics and engineering. There are many applications in engineering, electronics, seismic, identification composite materials, in the problems of non-destructive testing and etc., that entails intensive development of this direction.

Analysis of inverse spectral problems usually associated with the study of existence and uniqueness of solutions and development recovery algorithms for perturbing operator. As for the last of these questions, it is certainly worth mentioning the works of S.I. Kadchenko and his students, in which the method of regularized traces, and later, a new noniterative method for the numerical solution of inverse problems were proposed [1-4].

With regard to the existence and uniqueness of the solution of inverse problems, the number of jobs, in which the results for a specific operator, can be specified. For example, in the case of Laplace operator and degrees [5,6], but for the abstract self-adjoint operator such results for authors are unknown. This paper studies the existence of solution of the inverse spectral problem for abstract discrete self-adjoint operators with some conditions on the eigenvalues.

\section{The Existence Theorem of the Solution in the Inverse Spectral Problem}

Consider a discrete, self-adjoint, semibounded from below operator $T: L_{2}(\Omega) \rightarrow$ $L_{2}(\Omega)$, where $\Omega \subset \mathbb{R}^{2}$. Let the resolvent of this operator $R_{0}=(T-\lambda I)^{-1} \in \mathfrak{G}_{1}$, i.e. be a nuclear operator. Let operator $P$ be a limited the multiplication operator by complex-valued function $p \in L_{2}$. Let the spectrum of operator a simple. Denote by $\lambda_{n}$ eigenvalues of unperturbed operator $T$ numbered in the increasing order,$v_{n}$ - corresponding eigenfunctions. The eigenvalues $\mu_{n}$ of perturbed operator $T+P$ are determined by solving the operator equation

$$
T v+p v=\mu v
$$


with certain homogeneous boundary conditions. We pose the inverse problem - identify potential $p$ using eigenvalues $\mu_{n}$ and eigenfunctions $v_{n}$ of operator $T$ and eigenvalues of operator $T+P$.

Let us introduce some necessary notation. As $T$ is a discrete operator, then for any $\lambda_{n}$ exists $r_{n}$ such that $r_{n}=\frac{1}{2} \min _{n}\left\{\lambda_{n}-\lambda_{n-1} ; \lambda_{n+1}-\lambda_{n}\right\}, r_{0}=\inf _{n} r_{n}$. Define the contour $\gamma_{n}=\left\{\lambda \in \mathbb{C}:\left|\lambda_{n}-\lambda\right|=r_{n}\right\} . \Omega_{r_{n}}=\left\{\lambda:\left|\lambda_{n}-\lambda\right| \geq r_{0}\right\} ; \widetilde{\Omega}=\bigcap_{n=1}^{\infty} \Omega_{r_{n}}$. For $\lambda_{n}$ rightly

$$
\ldots \leq a_{t-1} \leq \lambda_{t}-r_{t}<\lambda_{t}<\lambda_{t}+r_{t} \leq a_{t} \leq \lambda_{t+1}-r_{t+1}<\lambda_{t+1}<\lambda_{t+1}+r_{t+1} \leq a_{t+1} \leq \ldots
$$

Lemma 1. If $\|P\|<r_{0}$ then $T+P$ is discrete, and if $R_{0} \in \mathfrak{G}_{1}$ then $R \in \mathfrak{G}_{1}$ and all root subspace of operator $T+P$ have the same dimension as the operator $T$.

Theorem 1. Let $\|P\|<r_{0}$, for any $n \in \mathbb{N}$ the spectral identity

$$
\mu_{n}=\mu_{n}+\left(P \varphi_{n}, \varphi_{n}\right)+\alpha_{n}
$$

where

$$
\alpha_{n}=\sum_{k=2}^{\infty} \frac{1}{2 \pi i} \int_{\gamma_{n}} \lambda\left[R_{0}(\lambda) P\right]^{k} R_{0}(\lambda) d \lambda
$$

is fulfilled.

It should be noted that in the proof of lemma 1 and theorem 1 authors follow the work [6].

Lemma 2. Let $n \gg 1, a_{n-1} \leq \operatorname{Re} \lambda \leq a_{n}$ we have the estimate:

$$
\sum_{m=1}^{\infty} \frac{1}{\left|\lambda-\lambda_{m}\right|^{2}} \leq \frac{1}{\left|\lambda-\lambda_{n}\right|^{2}}+\frac{1}{r_{n}^{2}}\left(2+\pi+\frac{1}{C^{1 / 2}}\right) .
$$

Proof.

$$
\begin{gathered}
\sum_{j=1}^{\infty} \frac{1}{\left|\lambda-\lambda_{j}\right|^{2}}<\frac{1}{\left|\lambda-\lambda_{n}\right|^{2}}+ \\
+\sum_{j=n+2}^{\infty} \frac{1}{\left(\lambda_{j}-a_{n}\right)^{2}}+\frac{1}{\left(a_{n-1}-\lambda_{n-1}\right)^{2}}+\frac{1}{\left(\lambda_{n+1}-a_{n}\right)^{2}}+\sum_{j=1}^{n-2} \frac{1}{\left(a_{n-1}-\lambda_{j}\right)^{2}} .
\end{gathered}
$$

For this $n$ there $k$ and $s$ such that $\lambda_{n}=\lambda_{k s}$. Denote $k^{2}+s^{2}=\widetilde{r}^{2}$.

$$
\begin{gathered}
\sum_{j=n+2}^{\infty} \frac{1}{\left(\lambda_{j}-a_{n}\right)^{2}}=\sum_{j=n+1}^{\infty} \frac{1}{\left(\lambda_{j}-a_{n-1}\right)^{2}}=\sum_{p^{2}+q^{2}>\widetilde{r}^{2}} \frac{1}{\left(\lambda_{p q}-a_{n-1}\right)^{2}}=\frac{\pi}{2} \int_{\widetilde{r}^{2}}^{\infty} \frac{\rho d \rho}{\left(\rho^{4}-a_{n-1}\right)^{2}} . \\
\frac{\pi}{2} \int_{\widetilde{r}^{2}}^{\infty} \frac{\rho d \rho}{\left(\rho^{4}-a_{n-1}\right)^{2}}=\frac{1}{a_{n-1} \widetilde{r}^{4}-a_{n-1}^{2}}=\frac{1}{a_{n-1}\left(\lambda_{n}-a_{n-1}\right)} \leq \frac{1}{a_{n-1} r_{n}} \leq \frac{1}{r_{n}^{2}} .
\end{gathered}
$$

We make the following assessment

$$
\sum_{j=1}^{n-2} \frac{1}{\left(a_{n-1}-\lambda_{j}\right)^{2}}=\sum_{j=1}^{n-1} \frac{1}{\left(a_{n}-\lambda_{j}\right)^{2}}=\sum_{j=1}^{n-1} \frac{1}{\left(a_{n}-\lambda_{p q}\right)^{2}}=
$$




$$
\begin{gathered}
=\sum_{\left(p^{2}+q^{2}\right)<\widetilde{r}^{2}} \frac{1}{\left(a_{n}-\left(p^{2}+q^{2}\right)^{2}\right)^{2}} \leq \int_{0}^{\widetilde{r}} \int_{0}^{\sqrt{\widetilde{r}^{2}-p^{2}}} \frac{d p d q}{\left(a_{t}-\left(p^{2}+q^{2}\right)^{2}\right)^{2}}= \\
=\frac{\pi}{2} \int_{0}^{\widetilde{r}} \frac{\rho d \rho}{\left(b_{n}-\rho^{4}\right)^{2}}=\frac{\pi}{2} \sum_{k=1}^{\infty} \frac{k}{a_{n}^{k+1}} \int_{0}^{\widetilde{r}} \frac{\rho^{4 k}}{\rho^{3}} d \rho= \\
=\frac{\pi}{2} \sum_{k=1}^{\infty} \frac{k}{a_{n}^{k+1}} \int_{0}^{\widetilde{r}} \rho^{4 k-5} d \rho=\frac{\pi}{2} \sum_{k=1}^{\infty} \frac{k}{a_{n}^{k+1}} \cdot \frac{\widetilde{r}^{4(k-1)+2}}{4(k-1)+2}< \\
<\frac{\pi}{2} \cdot \frac{\widetilde{r}^{2}}{a_{n}\left(a_{n}-\widetilde{r}^{4}\right)}=\frac{\pi}{2} \cdot \frac{\lambda_{n}^{1 / 2}}{\left(\lambda_{n}+r_{n}\right) r_{n}}<\frac{\pi}{2} \frac{\lambda_{n}^{1 / 2}}{\lambda_{n} r_{n}}=\frac{\pi}{2 \lambda_{n}^{1 / 2} r_{n}} .
\end{gathered}
$$

Since $n \gg 1$, then use the asymptotic behavior

$$
\frac{\pi}{2 \lambda_{n}^{1 / 2} r_{n}}=\frac{\pi}{2 C n r_{n}}=\frac{\pi \beta}{4 C n r_{n}}=\frac{\pi \beta}{2 r_{n}^{2}}
$$

In this way $\sum_{j=1}^{n-2} \frac{1}{\left(a_{n-1}-\lambda_{j}\right)^{2}}<\frac{\pi \beta}{2 r_{n}^{2}}$.

Putting all the estimates together, we obtain the required.

Theorem 2. Let $T$ be a discrete self-adjoint semibounded from below and $P$ is a bounded operators, acting in a separable Hilbert space $H$. If the spectrum of operator a simple, $\lambda_{n} \sim C n^{\beta}, \beta>1$, and the sequence $\mu_{n}^{k}$ of eigenvalues of operator $T+P$ is such that

$$
\sum_{n=1}^{\infty}\left|\mu_{n}-\lambda_{n}\right|<\frac{r}{2}(1-\omega)
$$

here $\omega=2 s r<1, s=\left(\sum_{n=1}^{\infty} r_{n}^{2}\left(\max _{\lambda \in \gamma_{n}}\left\|R_{0}(\lambda)\right\|_{2}^{2}\right)^{2}\right)^{1 / 2}, \quad r \in\left(0, \min \left\{r_{0}, \frac{1}{2 s}\right\}\right)$, then the solution $p \in L_{2}(\Omega)$ of inverse spectral problem exists.

Proof. In $L_{2}(\Omega)$ cosider the equation relatively $p$ :

$$
p=\alpha_{0}-\alpha(p), \alpha_{0}=\sum_{n=1}^{\infty}\left(\mu_{n}-\lambda_{n}\right) \varphi_{n}, \alpha(p)=\sum_{n=1}^{\infty} \alpha_{n}(p) \varphi_{n} .
$$

Introduce the operator $A: L_{2}(\Omega) \rightarrow L_{2}(\Omega)$, defined equality

$$
A p=\alpha_{0}-\alpha(p) .
$$

As

$$
\|A p\|_{L_{2}} \leq\left\|\alpha_{0}\right\|+\|\alpha(p)\| \leq \frac{r}{2}(1-\omega)+\frac{r}{2} \omega=\frac{r}{2},
$$

then operator $A$ displays a closed ball $U\left(0, \frac{r}{2}\right)$ at itself. It can be shown that the operator $A$ is a contractive operator. According to the Banach principle the equation has a unique solution $p$. We define the operator $P$, acting in $L_{2}(\Omega)$, as follows: $P \varphi(x)=p(x) \varphi(x)$, where $p$ - solution of equation (3). By direct substitution it can be shown, that $p$ is the desired potential. The theorem is proved. 


\section{References}

1. Kadchenko S.I. Numerical Method for the Solution of Inverse Problems Generated by Perturbations of Self-Adjoint Operators by Method of Regularized Traces. Vestnik of Samara State University, 2013, no. 6 (107), pp. 23-30. (in Russian)

2. Kadchenko S.I. A Numerical Method for Solving Inverse Problems Generated by the Perturbed Self-Adjoint Operators. Bulletin of the South Ural State University. Series: Mathematical Modelling, Programming and Computer Software, 2013, vol. 6, no. 4, pp. 15-25. (in Russian)

3. Kadchenko S.I. [Solution of Inverse Spectral Problems Generated by the Perturbed Self-Adjoint Operators by Method of Regularized Traces]. Bulletin of the Magnitogorsk State University. Mathematics, 2013, issue 15, pp. 34-43. (in Russian)

4. Kadchenko S.I. Numerical Method for Solving Inverse Spectral Problems Generated by Perturbed Self-Adjoint Operators. Vestnik of Samara State University, 2013, no. 91 (110), pp. 5-11. (in Russian)

5. Zakirova G.A., Sedov A.I. An Inverse Spectral Problem for Laplace Operator and it's Approximate Solution. Bulletin of the South Ural State University. Series: Mathematical Modelling, Programming and Computer Software, 2008, no. 27 (127), issue 2, pp. 19-27. (in Russian)

6. Zakirova G.A., Sedov A.I. An Inverse Problem for the Laplace Operator in the Isosceles Rectangular Triangle. Vestnik of Samara State University, 2008, no. 2, pp. 34-42. (in Russian)

Galiia A. Zakirova, candidate of physical and mathematical sciences, associate professor, department of Equations of Mathematical Physics, South Ural State University, Chelyabinsk, Russian Federation, zakirovaga@susu.ac.ru.

Evgenii $V$. Kirillov, postgraduate student, department of Equations of Mathematical Physics, South Ural State University, Chelyabinsk, Russian Federation, kirillovev@susu.ac.ru.

Received November 29, 2015

УДК 517.9

DOI: $10.14529 /$ jcem 150410

СУШЕСТВОВАНИЕ РЕШЕНИЯ ОБРАТНОЙ СПЕКТРАЛЬНОЙ ЗАДАЧИ ДЛЯ ДИСКРЕТНОГО САМОСОПРЯЖКННОГО ПОЛУОГРАНИЧЕННОГО СНИЗУ ОПЕРАТОРА

\section{Г.А. Закирова, Е.В. Кириллов}

Обратные спектральные задачи имеют много приложений в технике и физике. Исследовано множество задач для конкретных операторов. В данной статье исследуется обратная спектральная задача для абстрактного дискретного самосопряженного полуограниченного снизу оператора. При помощи резольвентного метода и принципа сжимающего отображения доказана теорема о существовании решения обратной задачи.

Ключевые слова: возмущенный оператор; дискретный самосопряљенный оператор; потенииал. 


\section{Литература}

1. Кадченко, С.И. Численный метод решения обратных задач, порожденных возмущенными самосопряженными операторами, методом регуляризованных следов / С.И. Кадченко // Вестник Самарского государственного университета. - 2013. № 6 (107). - С. 23-30.

2. Кадченко, С.И. Численный метод решения обратных задач, порожденных возмущенными самосопряженными операторами / С.И. Кадченко // Вестник ЮУрГУ. Серия: Математическое моделирование и программирование. - 2013. - Т. 6. № 4. - C. 15-25.

3. Кадченко, С.И. Численный метод решения обратных задач, порожденных возмущенными самосопряженными операторами, методом регуляризованных следов / С.И. Кадченко // Вестник МаГУ. Математика. - Вып. 15 - Магнитогорск: МаГУ, 2013. - C. 34-43.

4. Кадченко, С.И. Численный метод решения обратных задач, порожденных возмущенными самосопряженными операторами, методом регуляризованных следов / С.И. Кадченко // Вестник Самарского государственного университета. - 2013. № 9-1 (110). - С. 5-11.

5. Закирова, Г.А. Обратная спектральная задча для оператора Лапласа и ее приближенное решение / Г.А. Закирова, А.И. Седов // Вестник ЮУрГУ. Серия: Математическое моделирование и программирование. - 2008. - № 27 (127), вып. 2. C. $19-27$.

6. Закирова, Г.А. Обратная спектральная задача для оператора Лапласа на равнобедренном прямоугольном треугольнике / Г.А. Закирова, А.И. Седов // Вестник Самарского государственного университета. - 2008. - № 2. - С. 34-42.

Закирова Галия Амрулловна, кандидат физико-математических наук, доцент, кафедра уравнениий математической физики, Южно-Уральский государственный университет (г. Челябинск, Российская Федерация), zakirovaga@susu.ac.ru.

Кириллов Евгений Вадимович, аспирант, кафедра уравнений математической физики, Южно-Уральский государственный университет (ә. Челябинск, Российская Федераuия),kirillovev@susu.ac.ru.

Поступила в редакцию 29 ноября 2015 г. 